Supporting Information

\title{
pH Regulated Synthesis of Monodisperse Penta-twinned Gold Nanoparticles with High Yield
}

\author{
Xing Zhang, Reese Gallagher, Dong He, and Gang Chen*
}

\author{
AUTHOR INFORMATION \\ Corresponding Author \\ * Gang Chen - Department of Chemistry, University of Central Florida, Orlando, FL 32816 (USA); Email: gang.chen@ucf.edu
}

Authors

Xing Zhang - Department of Chemistry, University of Central Florida, Orlando, FL 32816 (USA)

Reese Gallagher - Department of Chemistry, University of Central Florida, Orlando, FL 32816 (USA)

Dong He - Department of Chemistry, University of Central Florida, Orlando, FL 32816 (USA); and State Key Laboratory on Integrated Optoelectronics, College of Electronic Science and Engineering, Jilin University, Changchun, Jilin 130012 (China) 


\section{Table of Contents}

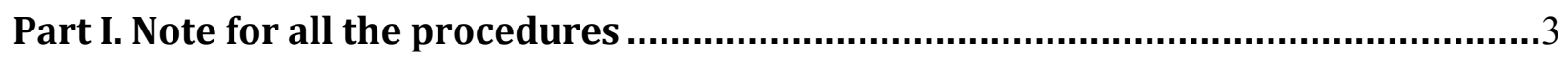

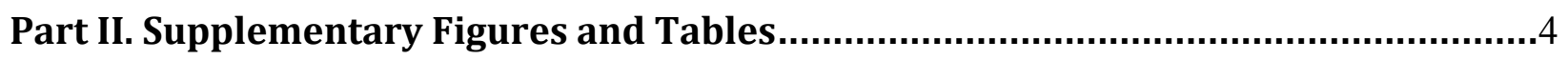

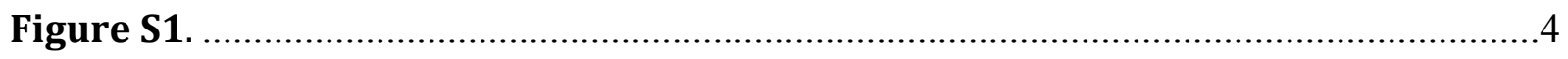

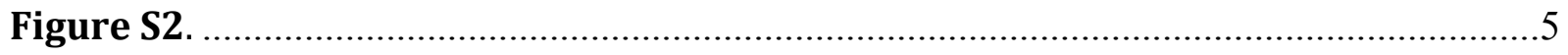

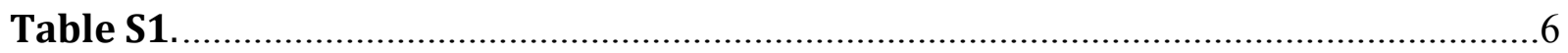

Table S2

Figure S3.

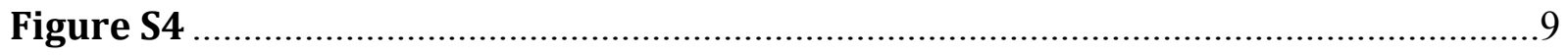

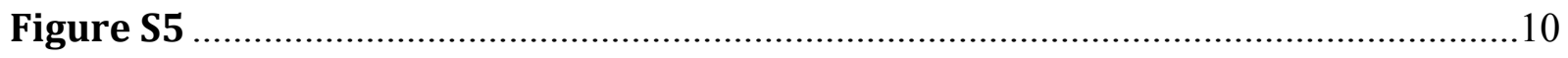

Table S3

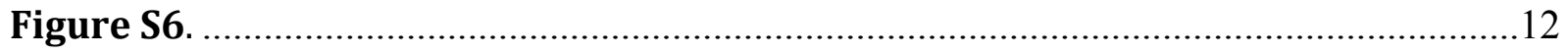

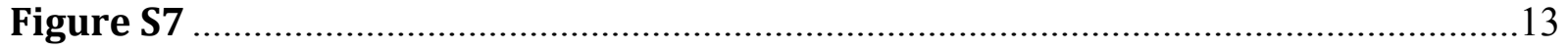

Table S4

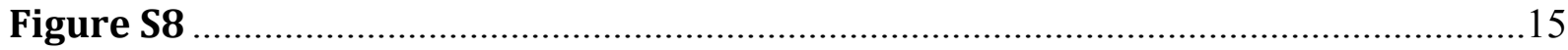

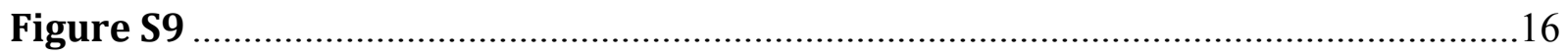

Table S5 


\section{Part I. Note for all the procedures}

All glassware was absolutely pristine. Any used glassware was washed with concentrated aqua regia (volume ratio of $\mathrm{HCl}: \mathrm{HNO}_{3}$ is 3:1) including stir bars. Before each synthesis, the glassware was washed with ultrapure water and dried via compressed air.

Reference $\mathrm{pH}$ solution: A reference solution was made to test the initial $\mathrm{pH}$ value of the growth solution for various amounts of acid or base added. The reason for using a reference as opposed to measuring the growth solution itself was to ensure that the growth solution came into contact with as little contamination as possible so as not to affect the seed-mediated synthesis. For the reference solution the same amount of $0.1 \mathrm{M} \mathrm{CTAB}, 0.1 \mathrm{M} \mathrm{HAuCl}_{4}, 10 \mathrm{mM} \mathrm{AgNO}_{3}$ and reductants was used as in the growth solution (here note: seeds were not used). At different amounts of acid or base the $\mathrm{pH}$ was measured (see corresponding tables for amounts and values). All measurements were made using a Thermo Scientific Orion Star A211 that was calibrated before use.

\section{List of abbreviation:}

LSPR - localized surface plasmon resonance

FWHM - full width at half maximum

TEM - transmission electron microscopy

UV-Vis - ultraviolet-visible

CTAB - hexadecyltrimethylammonium bromide 


\section{Part II. Supplementary Figures and Tables}

How to measure the size of bipyramid and decahedron

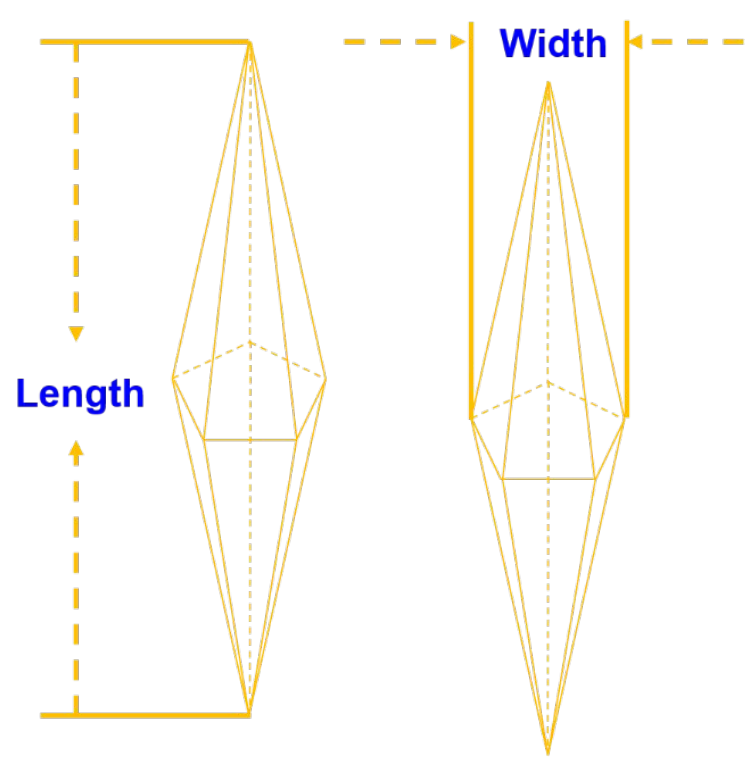

Bipyramid

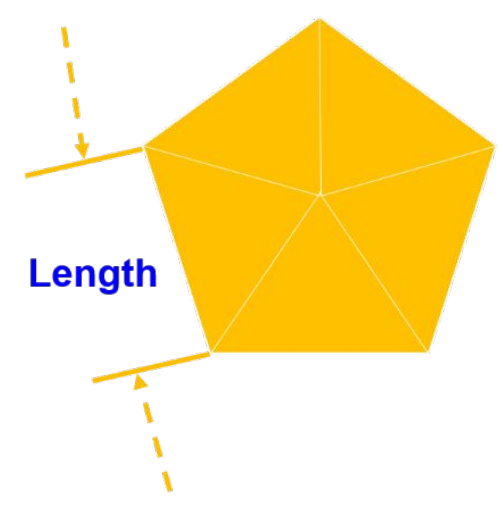

Decahedron

Figure S1. Schematic representation of the size measurement of bipyramid and decahedron.

The length of bipyramid is defined as the distance between its two apexes along the longitudinal axis, while its width is defined as the length of the projection of the pyramid base on the substrate. The length of decahedron is defined as its edge length. 


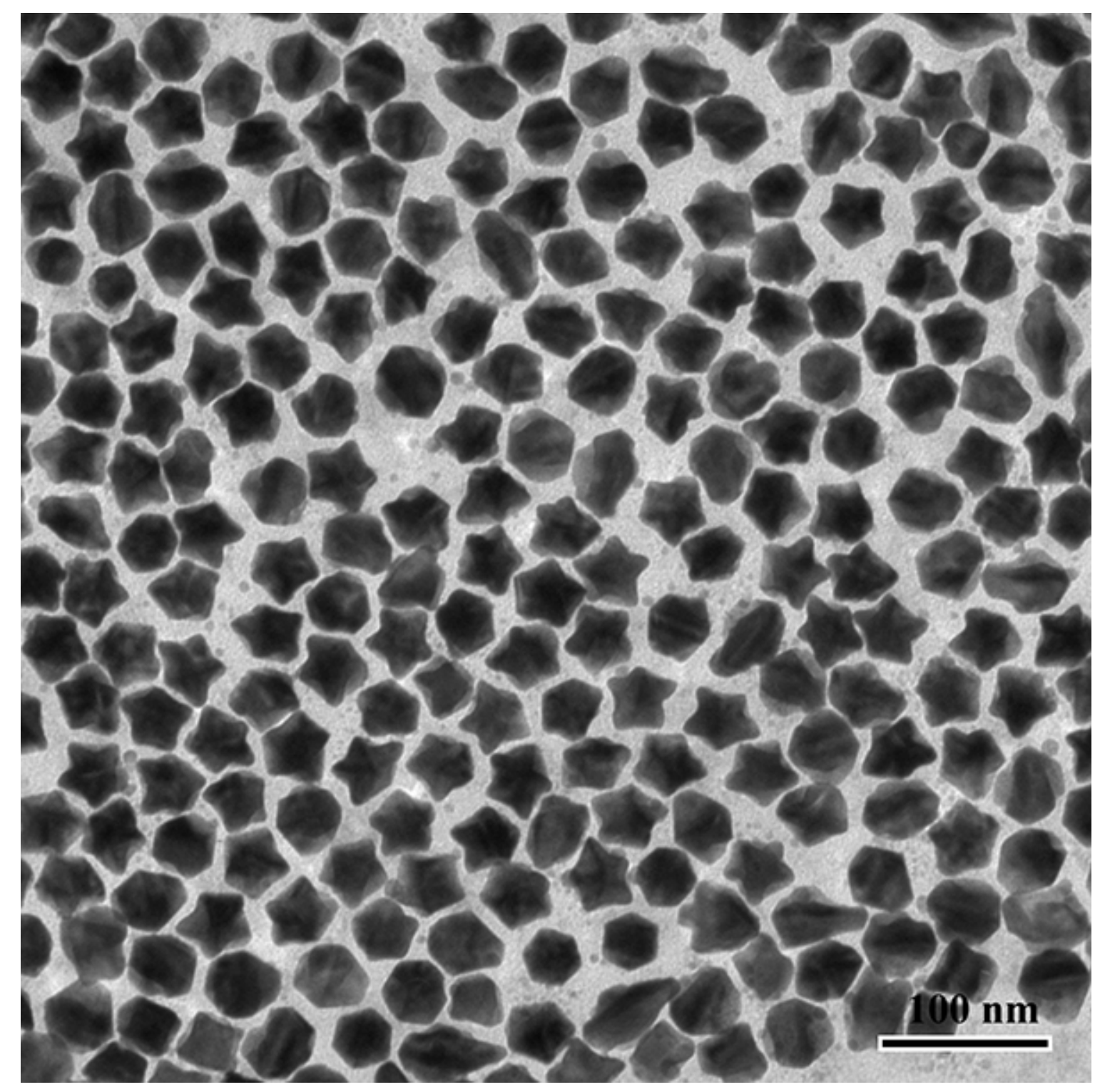

Figure S2. TEM image of the product with starfish shape prepared from ascorbic acid at pH 6.39 by adding $150 \mu \mathrm{L}$ of $\mathrm{NaHCO}_{3}\left(0.2 \mathrm{~mol} \cdot \mathrm{L}^{-1}\right)$ to the growth solution. 
Table S1. LSPR, FWHM, dimensions, and aspect ratios of the penta-twinned nanoparticles prepared from ascorbic acid at different $\mathrm{pH}$.

\begin{tabular}{ccccccc}
\hline LSPR (nm) & FWHM (nm) & Length (nm) & Width $(\mathrm{nm})$ & Aspect ratios & ${ }^{[\mathrm{a}]} \mathrm{HCl}(\mu \mathrm{L})$ & $\mathrm{pH}$ \\
\hline 601 & 63 & $47.1 \pm 3.4$ & $28.0 \pm 2.3$ & $1.69 \pm 0.14$ & 300 & 0.31 \\
636 & 60 & $56.7 \pm 3.7$ & $28.2 \pm 1.6$ & $2.01 \pm 0.13$ & 225 & 0.42 \\
695 & 66 & $63.5 \pm 4.4$ & $27.0 \pm 2.0$ & $2.36 \pm 0.14$ & 175 & 0.53 \\
727 & 60 & $67.5 \pm 4.1$ & $27.7 \pm 2.0$ & $2.44 \pm 0.13$ & 150 & 0.61 \\
750 & 59 & $68.6 \pm 4.2$ & $26.8 \pm 1.8$ & $2.57 \pm 0.14$ & 100 & 0.76 \\
773 & 60 & $71.2 \pm 4.3$ & $26.9 \pm 1.8$ & $2.65 \pm 0.17$ & 60 & 0.98 \\
809 & 60 & $78.3 \pm 3.7$ & $26.0 \pm 1.9$ & $3.01 \pm 0.19$ & 20 & 1.45 \\
\hline 635 & 68 & $78.9 \pm 3.6$ & $24.0 \pm 2.4$ & $3.32 \pm 0.32$ & 10 & 1.73 \\
\hline
\end{tabular}

[a] The concentration of $\mathrm{HCl}$ solution is $12.7 \mathrm{~mol} \cdot \mathrm{L}^{-1}$ 
Table S2. LSPR, FWHM, dimensions, and aspect ratios of the penta-twinned nanoparticles prepared from hydroquinone at different $\mathrm{pH}^{[\mathrm{a}]}$

\begin{tabular}{|c|c|c|c|c|c|c|}
\hline LSPR (nm) & FWHM (nm) & Length (nm) & Width (nm) & Aspect ratios & ${ }^{[\mathrm{b}]} \mathrm{NaHCO}_{3}(\mu \mathrm{L})$ & $\mathrm{pH}$ \\
\hline 570 & [d] 57 & $37.4 \pm 3.0$ & $26.1 \pm 1.8$ & $1.44 \pm 0.13$ & 40 & 3.11 \\
\hline 605 & [d] 62 & $52.1 \pm 4.7$ & $30.1 \pm 1.8$ & $1.74 \pm 0.17$ & 60 & 3.42 \\
\hline 640 & 77 & $56.8 \pm 4.7$ & $28.8 \pm 1.5$ & $1.97 \pm 0.15$ & 80 & 4.37 \\
\hline 665 & 81 & $65.4 \pm 4.0$ & $29.5 \pm 1.1$ & $2.22 \pm 0.12$ & 85 & 5.27 \\
\hline 705 & 99 & $68.3 \pm 4.2$ & $28.0 \pm 1.1$ & $2.44 \pm 0.16$ & 88 & 5.39 \\
\hline 745 & 86 & $77.8 \pm 3.8$ & $27.8 \pm 2.0$ & $2.81 \pm 0.19$ & 90 & 5.45 \\
\hline 780 & 54 & $77.8 \pm 2.0$ & $26.8 \pm 1.2$ & $2.91 \pm 0.14$ & 95 & 5.60 \\
\hline 800 & 71 & $82.9 \pm 3.2$ & $26.9 \pm 2.3$ & $3.11 \pm 0.32$ & 110 & 5.88 \\
\hline / & / & / & / & / & 120 & 5.96 \\
\hline / & / & / & / & / & 140 & 6.18 \\
\hline / & / & / & / & / & 160 & 6.34 \\
\hline [c] 575 & 93 & / & $46.4 \pm 3.5$ & / & 200 & 6.55 \\
\hline
\end{tabular}

[a] Some sample data are not available because of the irregular shape of the product or because the product is a mixture.

[b] The concentration of $\mathrm{NaHCO}_{3}$ solution is $0.2 \mathrm{~mol} \cdot \mathrm{L}^{-1}$.

[c] Decahedra were formed at this reaction condition.

[d] The data are derived from the curve fitting results. 


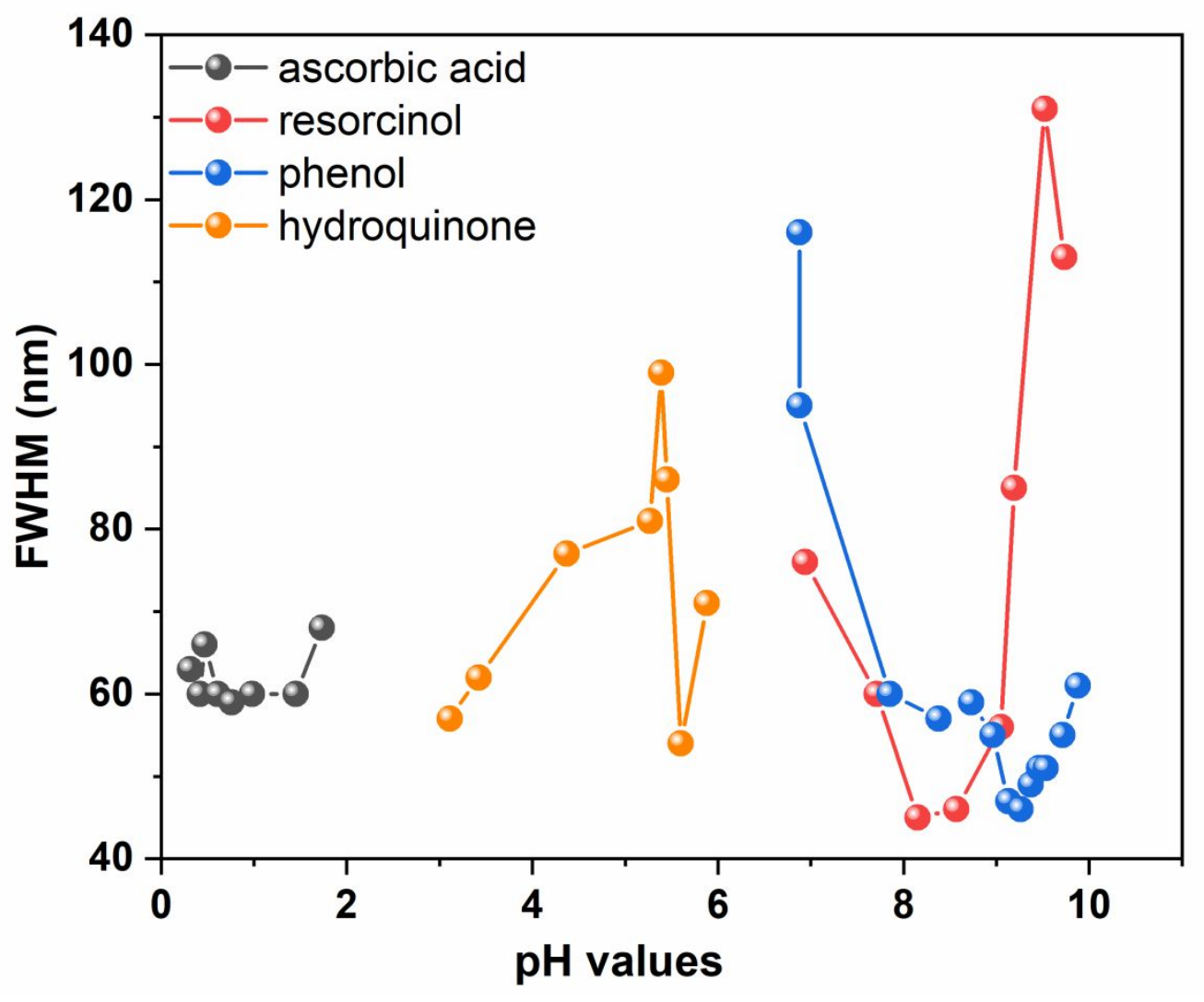

Figure S3. FWHM of the LSPR peaks of the penta-twinned nanoparticles synthesized from different reductants vs the pH values of the growth solution. 


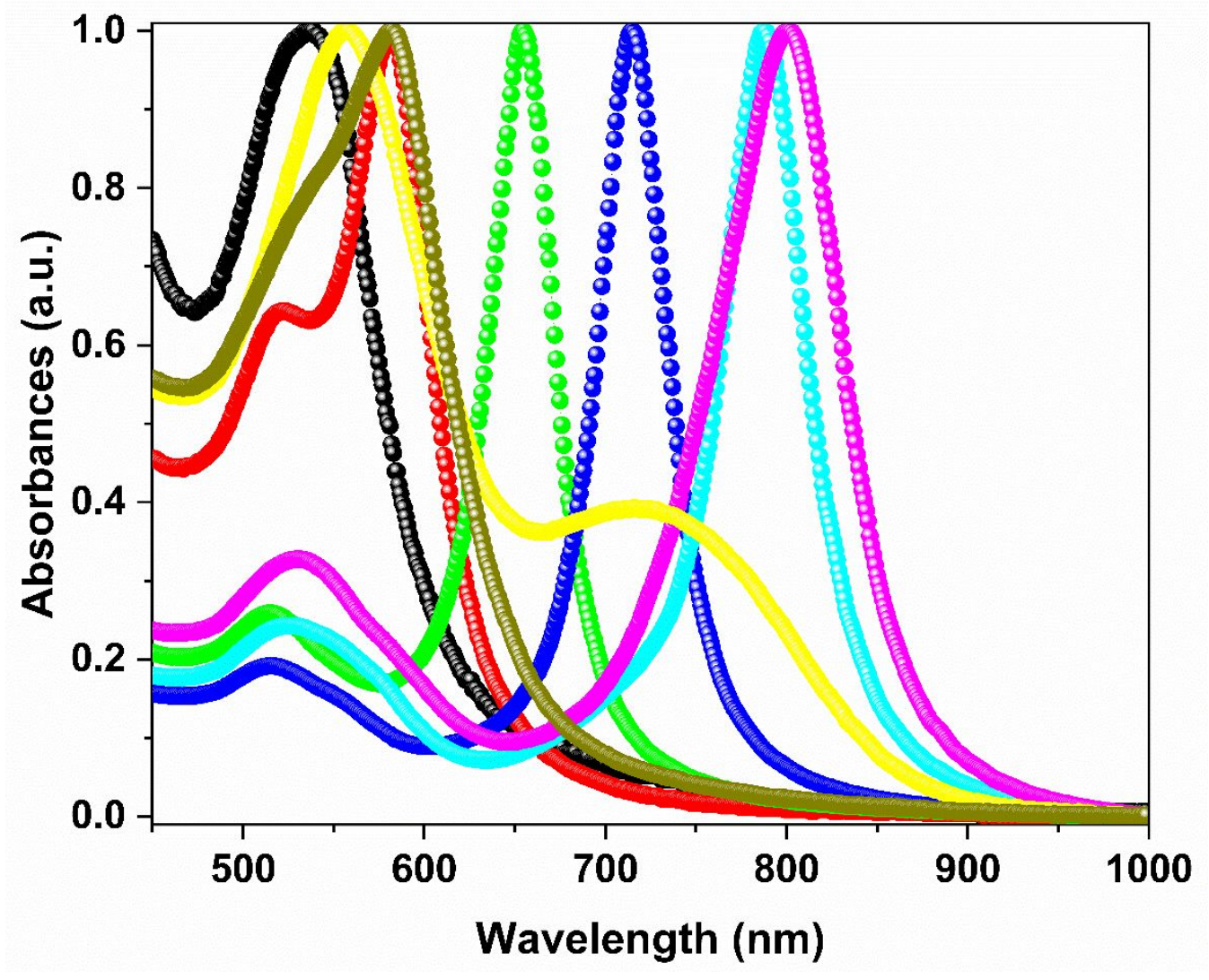

Figure S4. UV-Vis spectra of penta-twinned nanoparticles prepared from resorcinol at different pH: 6.94 (black), 7.71 (red), 8.15 (green), 8.57 (blue), 9.05 (cyan), 9.19 (magenta), 9.52 (yellow), and 9.73 (dark yellow). 

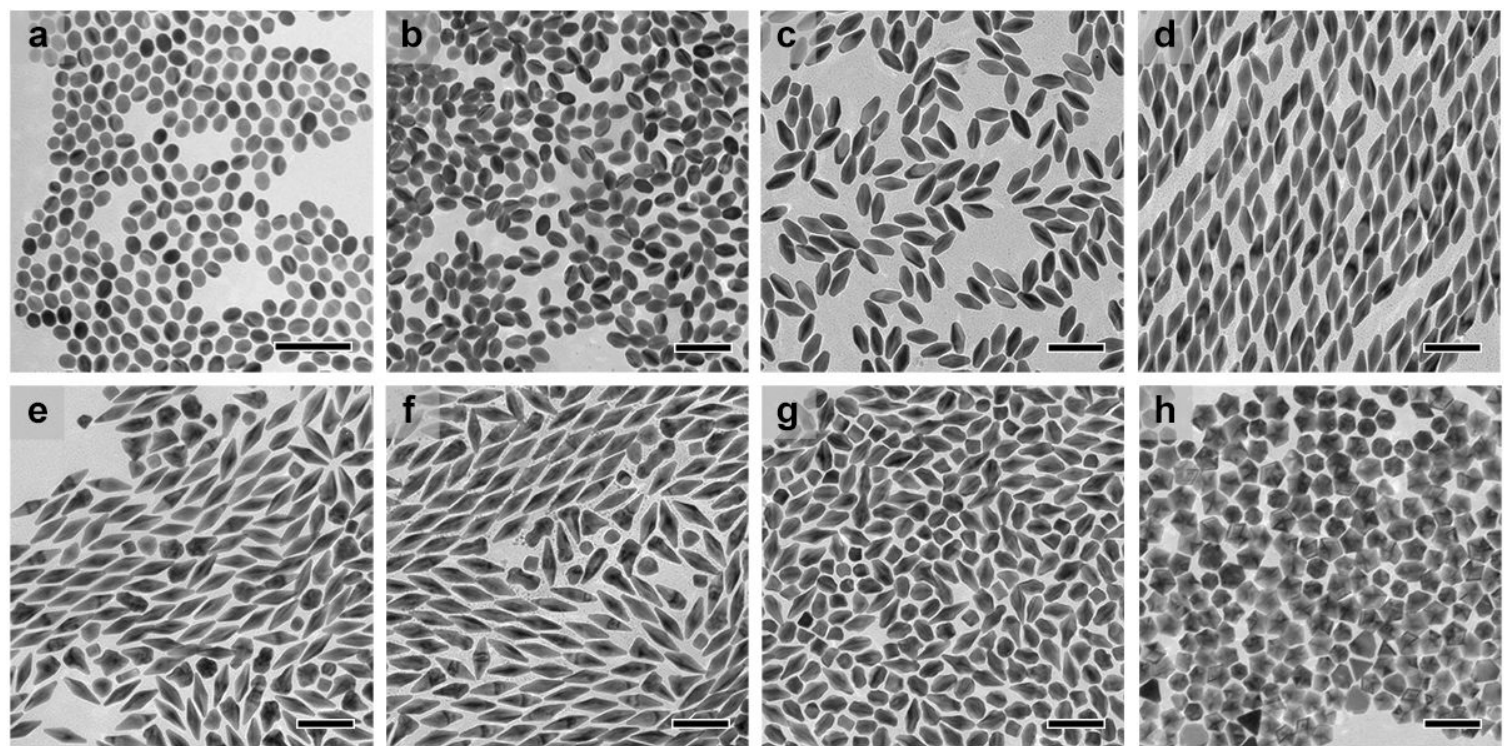

Figure S5. TEM images of penta-twinned nanoparticles prepared from resorcinol at different $\mathrm{pH}$. (a-h) the $\mathrm{pH}$ values of the growth solutions are 6.94, 7.71, 8.15, 8.57, 9.05, 9.19, 9.52, and 9.73, respectively. All scale bars are $100 \mathrm{~nm}$. 
Table S3. LSPR, FWHM, dimensions, and aspect ratios of the penta-twinned nanoparticles prepared from resorcinol at different $\mathrm{pH} .{ }^{[a]}$

\begin{tabular}{ccccccc}
\hline LSPR $(\mathrm{nm})$ & FWHM $(\mathrm{nm})$ & Length $(\mathrm{nm})$ & Width $(\mathrm{nm})$ & Aspect ratios & {$[\mathrm{b}] \mathrm{Na}_{2} \mathrm{CO}_{3}(\mu \mathrm{L})$} & $\mathrm{pH}$ \\
\hline 535 & [d] 76 & $24.8 \pm 1.4$ & $18.9 \pm 1.1$ & $1.31 \pm 0.06$ & 55 & 6.94 \\
581 & ${ }^{[\mathrm{d}]} 60$ & $36.3 \pm 1.6$ & $24.8 \pm 1.3$ & $1.46 \pm 0.08$ & 70 & 7.71 \\
654 & 45 & $56.4 \pm 2.7$ & $26.5 \pm 1.7$ & $2.13 \pm 0.12$ & 80 & 8.15 \\
715 & 46 & $63.8 \pm 2.0$ & $25.4 \pm 1.1$ & $2.51 \pm 0.13$ & 90 & 8.57 \\
786 & 56 & $74.2 \pm 3.2$ & $24.3 \pm 1.7$ & $3.07 \pm 0.26$ & 110 & 9.05 \\
801 & 85 & $75.9 \pm 3.8$ & $25.9 \pm 2.3$ & $2.96 \pm 0.32$ & 120 & 9.19 \\
722 & {$[\mathrm{~d}] 131$} & $/$ & $/$ & $/$ & 150 & 9.52 \\
[c] 582 & [d] 113 & $/$ & $40.9 \pm 3.95$ & $/$ & 180 & 9.73 \\
\hline
\end{tabular}

[a] Some sample data are not available because of the irregular shape of the product or because the product is a mixture.

[b] The concentration of $\mathrm{Na}_{2} \mathrm{CO}_{3}$ solution is $0.2 \mathrm{~mol} \cdot \mathrm{L}^{-1}$.

[c] Decahedra were formed at this reaction condition.

[d] The data are derived from the curve fitting results. 


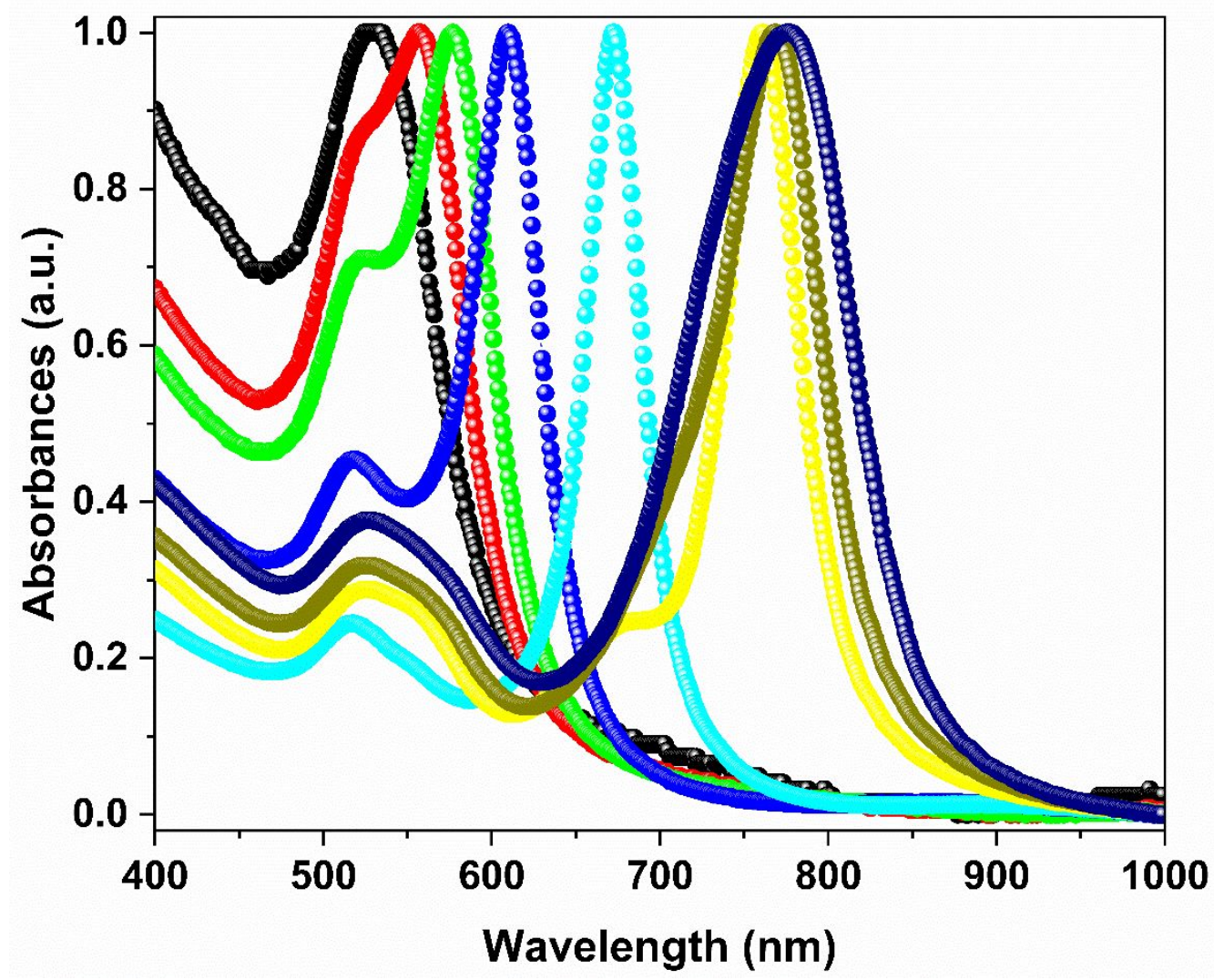

Figure S6. UV-Vis spectra of penta-twinned nanoparticles prepared from phloroglucinol at different pH: 6.68 (black), 7.06 (red), 7.41 (green), 7.70 (blue), 7.93 (cyan), 8.18 (yellow), 8.36 (dark yellow), and 8.49 (navy). 

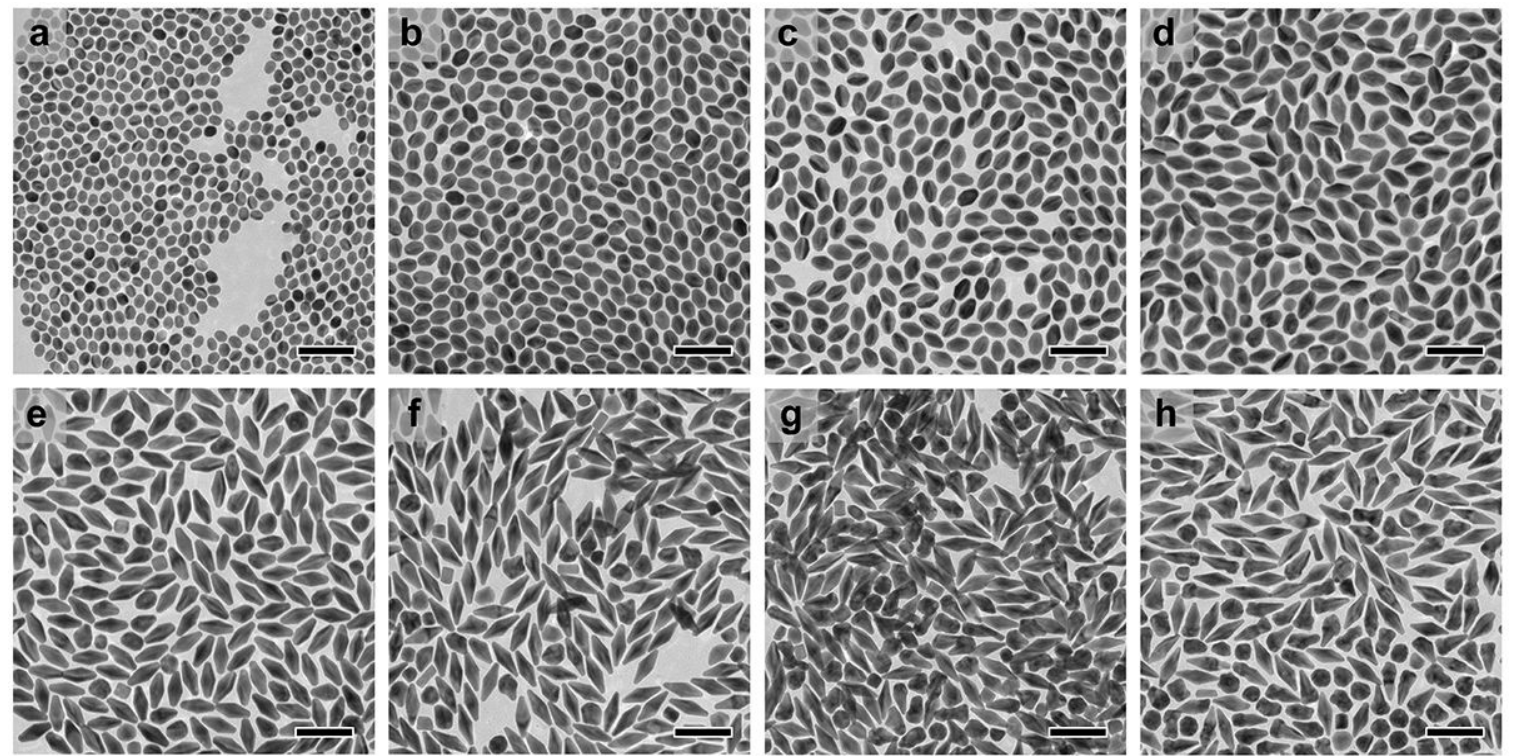

Figure S7. TEM images of penta-twinned nanoparticles prepared from phloroglucinol at different $\mathrm{pH}$. (a-h) the pH values of the growth solutions are 6.68, 7.06, 7.41, 7.70, 7.93, 8.18, 8.36, and 8.49, respectively. All scale bars are $100 \mathrm{~nm}$. 
Table S4. LSPR, FWHM, dimensions, and aspect ratios of the penta-twinned nanoparticles prepared from phloroglucinol at different $\mathrm{pH}$.

\begin{tabular}{ccccccc}
\hline LSPR (nm) & FWHM (nm) & Length (nm) & Width (nm) & Aspect ratios & ${ }^{[a]} \mathrm{Na}_{2} \mathrm{CO}_{3}(\mu \mathrm{L})$ & $\mathrm{pH}$ \\
\hline 530 & {$[\mathrm{~b}] 90$} & $23.8 \pm 1.1$ & $18.6 \pm 1.2$ & $1.28 \pm 0.07$ & 50 & 6.68 \\
563 & {$\left[{ }^{\mathrm{b}]} 62\right.$} & $35.4 \pm 1.7$ & $26.0 \pm 1.0$ & $1.36 \pm 0.06$ & 60 & 7.06 \\
578 & {$[\mathrm{~b}] 57$} & $40.2 \pm 2.0$ & $26.8 \pm 0.9$ & $1.50 \pm 0.07$ & 70 & 7.41 \\
610 & 57 & $49.2 \pm 1.7$ & $28.1 \pm 0.6$ & $1.74 \pm 0.07$ & 80 & 7.70 \\
673 & 42 & $60.9 \pm 1.4$ & $26.4 \pm 1.5$ & $2.31 \pm 0.11$ & 90 & 7.93 \\
761 & 56 & $68.7 \pm 2.6$ & $24.2 \pm 1.3$ & $2.85 \pm 0.17$ & 110 & 8.18 \\
769 & 82 & $71.1 \pm 1.7$ & $24.8 \pm 1.9$ & $2.88 \pm 0.22$ & 120 & 8.36 \\
778 & 114 & $71.0 \pm 2.2$ & $25.3 \pm 1.4$ & $2.82 \pm 0.20$ & 130 & 8.49 \\
\hline
\end{tabular}

[a] The concentration of $\mathrm{Na}_{2} \mathrm{CO}_{3}$ solution is $0.2 \mathrm{~mol} \cdot \mathrm{L}^{-1}$.

[b] The data are derived from the curve fitting results. 


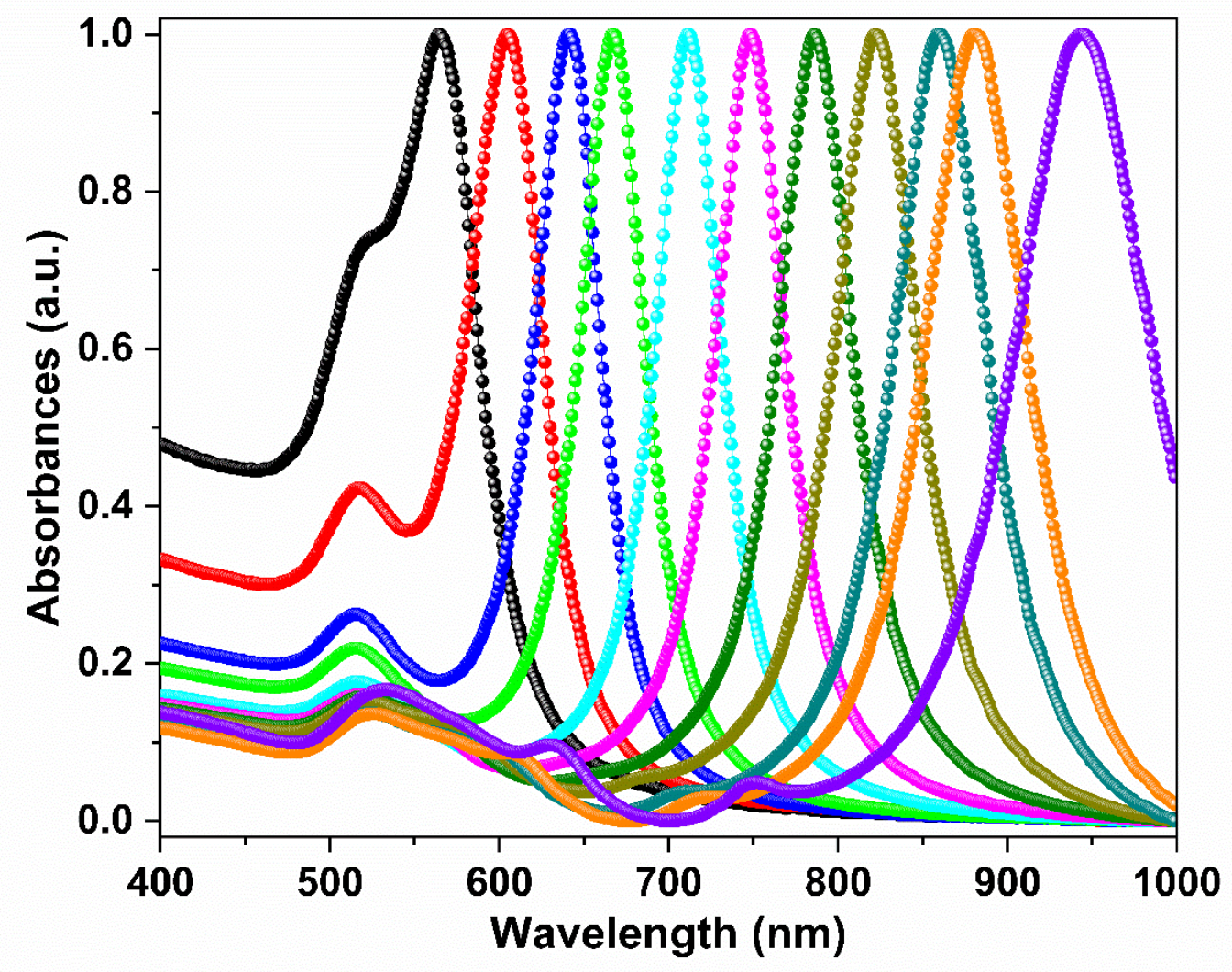

Figure S8. UV-Vis spectra of penta-twinned nanoparticles prepared from phenol with different amount of seeds. The corresponding volume of seeds are: $1.0 \mathrm{~mL}$ (black), $0.75 \mathrm{~mL}$ (red), $0.65 \mathrm{~mL}$ (blue), $0.6 \mathrm{~mL}$ (green), $0.5 \mathrm{~mL}$ (cyan), $0.4 \mathrm{~mL}$ (magenta), $0.25 \mathrm{~mL}$ (olive), $0.18 \mathrm{~mL}$ (dark yellow), $0.125 \mathrm{~mL}$ (dark cyan), $0.1 \mathrm{~mL}$ (orange), and $0.06 \mathrm{~mL}$ (violet). 

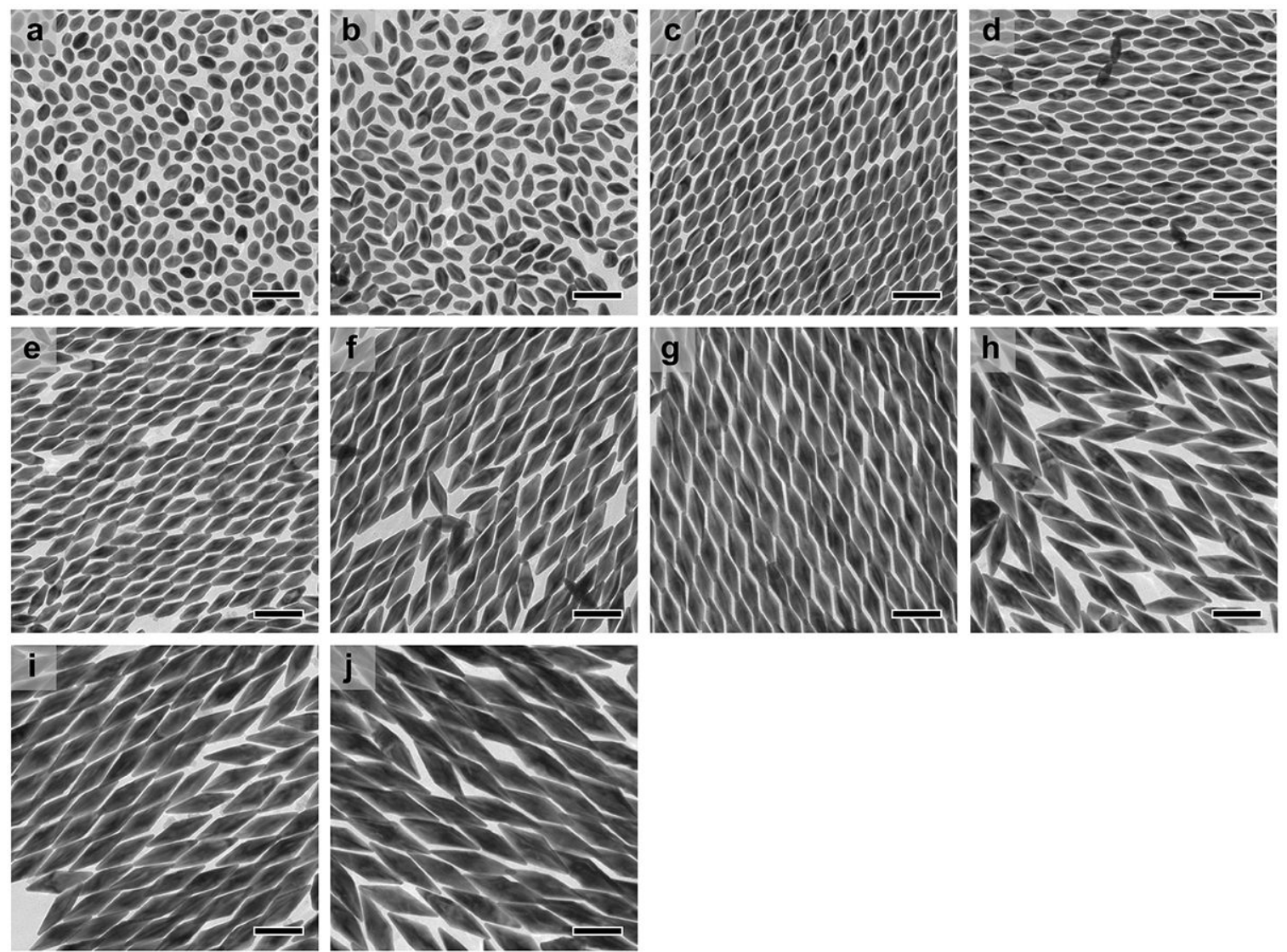

Figure S9. TEM images of penta-twinned nanoparticles prepared from phenol with different amount of seeds. (a-j) The volume of seeds are $1.0 \mathrm{~mL}, 0.75 \mathrm{~mL}, 0.65 \mathrm{~mL}, 0.6 \mathrm{~mL}, 0.5 \mathrm{~mL}, 0.25 \mathrm{~mL}, 0.18 \mathrm{~mL}, 0.125 \mathrm{~mL}, 0.1 \mathrm{~mL}$ and $0.06 \mathrm{~mL}$, respectively. All scale bars are $100 \mathrm{~nm}$ 
Table S5. LSPR, FWHM, dimensions, and aspect ratios of the penta-twinned nanoparticles prepared from phenol with different amount of seeds. ${ }^{[a]}$

\begin{tabular}{cccccc}
\hline LSPR (nm) & FWHM (nm) & Length (nm) & Width (nm) & Aspect ratios & Seeds (mL) \\
\hline 567 & {$[\mathrm{~b}] 58$} & $39.1 \pm 3.4$ & $26.1 \pm 1.8$ & $1.50 \pm 0.08$ & 1.00 \\
604 & 58 & $47.4 \pm 3.4$ & $26.2 \pm 1.5$ & $1.81 \pm 0.10$ & 0.75 \\
642 & 49 & $53.3 \pm 2.9$ & $25.4 \pm 1.6$ & $2.10 \pm 0.11$ & 0.65 \\
667 & 47 & $57.7 \pm 3.0$ & $25.5 \pm 1.5$ & $2.26 \pm 0.12$ & 0.60 \\
710 & 49 & $67.9 \pm 3.1$ & $26.0 \pm 1.5$ & $2.61 \pm 0.13$ & 0.50 \\
786 & 53 & $95.8 \pm 2.8$ & $32.3 \pm 2.0$ & $2.89 \pm 0.16$ & 0.25 \\
822 & 59 & $106.7 \pm 3.1$ & $34.2 \pm 2.0$ & $3.12 \pm 0.16$ & 0.18 \\
858 & 65 & $130.4 \pm 3.2$ & $39.3 \pm 2.4$ & $3.33 \pm 0.19$ & 0.125 \\
880 & 72 & $144.4 \pm 4.9$ & $43.9 \pm 4.2$ & $3.32 \pm 0.31$ & 0.10 \\
945 & 97 & $174.8 \pm 5.9$ & $46.8 \pm 3.1$ & $3.75 \pm 0.29$ & 0.06 \\
\hline
\end{tabular}

[a] Growth conditions

\begin{tabular}{ccc}
\hline Chemicals & Concentration $(\mathrm{mM})$ & Volume \\
\hline $\mathrm{CTAB}$ & 100 & $10 \mathrm{~mL}$ \\
$\mathrm{HAuCl}_{4}$ & 100 & $50 \mu \mathrm{L}$ \\
$\mathrm{AgNO}_{3}$ & 10 & $200 \mu \mathrm{L}$ \\
$\mathrm{Na}_{2} \mathrm{CO}_{3}$ & 200 & $140 \mu \mathrm{L}$ \\
Phenol & 100 & $150 \mu \mathrm{L}$ \\
Seeds & $/$ & various amounts of seeds \\
\hline
\end{tabular}

[b] The data is derived from the curve fitting results. 